CARNETS DE Carnets de géographes

GÉOGRAPHES.

5 | 2013

Géographie humanimale

\title{
Le rôle du chemin de fer en Afrique
}

Les cas du Mozambique et du Kenya

\section{Chantal Béranger}

\section{(2) OpenEdition}

Journals

Édition électronique

URL : http://journals.openedition.org/cdg/1092

DOI : $10.4000 /$ cdg. 1092

ISSN : 2107-7266

Éditeur

UMR 245 - CESSMA

Référence électronique

Chantal Béranger, "Le rôle du chemin de fer en Afrique », Carnets de géographes [En ligne], 5 | 2013, mis en ligne le 01 janvier 2013, consulté le 24 septembre 2020. URL : http://journals.openedition.org/ cdg/1092 ; DOI : https://doi.org/10.4000/cdg.1092

\section{(c) (i) $\odot$}

La revue Carnets de géographes est mise à disposition selon les termes de la Licence Creative Commons Attribution - Pas d'Utilisation Commerciale - Pas de Modification 4.0 International. 


\section{LE RÔLE DU CHEMIN DE FER EN AFRIQUE Les cas du Mozambique et du Kenya}

CHANTAL BERANGER

Cette thèse se situe dans l'articulation entre les thématiques du transport et du développement durable, par le biais du concept de congruence. Elle aborde plus spécifiquement la question du rôle du chemin de fer dans l'amélioration de la vie des populations rurales et particulièrement dans deux états africains, le Mozambique et le Kenya. La question était de savoir, dans quelle mesure le transport offre des circulations qui répondent aux attentes des populations, puisque nous avons la conviction que le chemin de fer peut contribuer à des dynamiques socio-économiques dans les zones rurales qu'il traverse.

L'objectif a été de comprendre vers quel type d'articulation des modes de transport, il faudrait s'orienter, pour un aménagement du territoire conciliant un développement social, économique et environnemental durable dans le temps, pour l'ensemble des populations et des entreprises. La démarche scientifique employée a été d'abord de faire un état de la question du XIXè au début du XXI ${ }^{\grave{e}}$ siècle par une recherche bibliographique et de statistiques tant en France qu'en Afrique. Un colloque international a été organisé pour compléter l'état de la question par des communications récentes. Dans le même temps, des observations et des entretiens ont pu être effectués sur le terrain, au Mozambique et au Kenya. Nous avons notamment recueilli la demande et l'expérience de la population.

Ces éléments ont conduit à une introduction théorique établissant l'environnement conceptuel. Nos analyses ont porté notre choix sur la circulation, l'aménagement du territoire et le développement durable, en utilisant trois éléments principaux. II s'agit des échelles, de la congruence et des coûts. Les relations campagne-ville ont été étudiées sous l'angle des solidarités et des relations entre les ruraux et les urbains.

La première partie traite du contexte et de l'état du chemin de fer africain. Ce contexte participe aux interrogations sur le développement durable. Une mise en relation des principaux éléments permet d'établir un modèle global de congruence négative. C'est-à-dire d'un processus qui conduit à la pauvreté. Cette partie permet de mettre en perspective les deux cas.

La seconde partie permet de décrire et d'analyser les deux cas très différents, du Mozambique et du Kenya. 
- au Mozambique depuis la remise en service de la ligne de Nacala fin 1992, jusqu'à sa privatisation en 2003 ;

- au Kenya, de 1995 où une interdiction de porter des petites marchandises aux petites gares a été instaurée, jusqu'à la privatisation de la ligne de Mombasa en 2005.

Cette partie repose sur la mise en valeur du cas de la ligne de Nacala. Les analyses aboutissent à la construction d'un modèle qui autorise une troisième partie prospective.

La troisième partie interroge sur le chemin de fer comme instrument du développement durable et sur les éléments complémentaires nécessaires. Nous nous attachons à proposer des apports pour une organisation de l'espace, dans un couloir de développement traversé par un chemin de fer, sans route parallèle.

La comparaison des cas du Mozambique et du Kenya a démontré que la complémentarité rail-route pouvait être à la base d'un processus pouvant conduire au développement durable pour les populations rurales, dans un couloir de développement. A contrario, la concurrence rail-route, la fermeture des gares ou de lignes ferroviaires, et la suppression de trains mixtes omnibus sont négatives pour la vie socio-économique des ruraux. Ces éléments négatifs contribuent à l'augmentation du prix du panier de la ménagère. En revanche, le chemin de fer peut permettre une répartition de la population tout au long de la ligne de façon linéaire, faciliter l'aménagement du territoire et en réduire le coût, tout autant que participer à la réduction de l'effet de serre. Le train facilite la vie socio-économique des ruraux. II permet l'organisation des sociétés et des dynamiques spatiales qui contribuent aux avantages comparatifs nécessaires à l'abaissement des coûts. II concoure au processus conduisant au développement durable. Le rôle du chemin de fer se pose comme décisif dans la gouvernance.

\section{Fiche informative}

\section{Lien électronique}

https://sites.google.com/site/berangergeographie/

\section{Discipline}

Géographie

\section{Directeur}

Benjamin Steck

\section{Université}

Université du Havre

Membres du jury de thèse, soutenue le 12 octobre 2012

- Bernard Calas, Professeur, Université de Bordeaux 3

- Bernard Charlery de la Masselière, Professeur, Université de Toulouse

- Benjamin Steck, Professeur, Université du Havre

- Jean Varlet, Professeur, Université de Savoie - Chambéry

- Pierre Zembri, Professeur, Université de Cergy-Pontoise

\section{Courriel de l'auteur} berangerc191@gmail.com 